ARTICLE

DOI: $10.1038 / \mathrm{s} 41467-018-05205-5$

\title{
Highly active enzymes by automated combinatorial backbone assembly and sequence design
}

Gideon Lapidoth', Olga Khersonsky', Rosalie Lipsh', Orly Dym², Shira Albeck ${ }^{2}$, Shelly Rogotner ${ }^{2}$ \& Sarel J. Fleishman (1) ${ }^{1}$

Automated design of enzymes with wild-type-like catalytic properties has been a longstanding but elusive goal. Here, we present a general, automated method for enzyme design through combinatorial backbone assembly. Starting from a set of homologous yet structurally diverse enzyme structures, the method assembles new backbone combinations and uses Rosetta to optimize the amino acid sequence, while conserving key catalytic residues. We apply this method to two unrelated enzyme families with TIM-barrel folds, glycoside hydrolase 10 (GH10) xylanases and phosphotriesterase-like lactonases (PLLs), designing 43 and 34 proteins, respectively. Twenty-one $\mathrm{GH} 10$ and seven PLL designs are active, including designs derived from templates with $<25 \%$ sequence identity. Moreover, four designs are as active as natural enzymes in these families. Atomic accuracy in a high-activity GH10 design is further confirmed by crystallographic analysis. Thus, combinatorial-backbone assembly and design may be used to generate stable, active, and structurally diverse enzymes with altered selectivity or activity.

\footnotetext{
${ }^{1}$ Department of Biomolecular Sciences, Weizmann Institute of Science, 7610001 Rehovot, Israel. ${ }^{2}$ Israel Structural Proteomics Center, Weizmann Institute of Science, 7610001 Rehovot, Israel. These authors contributed equally: Gideon Lapidoth, Olga Khersonsky. Correspondence and requests for materials should be addressed to S.J.F. (email: sarel@weizmann.ac.il)
} 
E nzymes can be grouped into families, members of which catalyze nearly identical chemical reactions, but exhibit vast differences in rates and substrate selectivities ${ }^{1-3}$. Conservation of chemical reactivity and diversity in substrate recognition are encoded in a modular architecture, wherein the residues actively taking part in catalysis are conserved in sequence and structure, typically including minute structural details. By contrast, structural elements outside the catalytic core vary substantially, including through insertion and deletion of large protein segments, to encode different substrate selectivities.

Enzymes belonging to the TIM-barrel fold, which is represented in five of the six top-level classes defined by the Enzyme Commission $(\mathrm{EC})^{3,4}$, are a prime example for this modularity. In each TIM-barrel family, eight parallel $\beta$-strands are arranged in a conserved and concentric barrel around the active-site pocket; the a-helices surround the strands and stabilize the pocket. By contrast to the atomic conservation of the catalytic residues in each family, the loops connecting the $\beta$-strands to the $\alpha$-helices are highly variable in length, conformation, and sequence; substrate selectivity is largely encoded in these variable regions. Owing to this structural modularity, new substrate selectivities can evolve through gene recombination among homologous TIM barrels followed by insertion, deletion, and mutation; that is, as long as the scaffold's structural stability and the geometry of the core catalytic residues are maintained, the loop regions can vary substantially ${ }^{5-7}$. Indeed, more than 70 distinct sequence families in the Structural Classification of Proteins (SCOP) belong to the TIM-barrel fold ${ }^{4,8}$, demonstrating how modularity has been exploited time and again by evolution. Structural modularity is a hallmark of other versatile enzyme classes, including, for instance, enzymes of the $\beta$-propeller, $\beta$-trefoil, Rossman, $\alpha / \alpha$-barrel, and $\alpha /$ $\beta$-hydrolase folds?.

Modularity has also been exploited to optimize enzymes through laboratory evolution and structure-based recombination ${ }^{10-12}$. For instance, laboratory genetic recombination among naturally occurring enzymes through structurally conserved sites has generated enzymes with large variations in stability and specific activity $^{13-18}$.

Structure-based recombination has also been used to fuse TIMbarrel fragments and even fragments from unrelated folds, to generate new structures ${ }^{19-22}$. These and other structure-based and computational design studies ${ }^{23-25}$ highlighted the structural adaptability of TIM barrels, but the resulting proteins were inactive, and in some cases, iterative laboratory evolution was employed, resulting in activities that were still several orders of magnitude lower than those of the wild type $\mathrm{e}^{18,22,26,27}$. Furthermore, de novo enzyme design, whereby constellations of up to four catalytic residues are installed on natural scaffold proteins that do not exhibit the desired activity, targeted elementary reactions and has resulted in marginally stable proteins and catalytic efficiencies that were orders of magnitude lower than those of natural enzymes ${ }^{28-30}$, similarly requiring iterative laboratory evolution to improve stability and rates and to obtain the designed active-site constellation ${ }^{31-33}$. Thus, automated design of stable and sophisticated enzymes exhibiting catalytic efficiencies that rival those of natural ones has been a long-standing though elusive goal $^{34-36}$.

Here, we demonstrate a path to automated design of stable and highly active enzymes. The design method is inspired by the evolution of new enzymes in nature through recombination, insertion, deletion, and mutation ${ }^{37,38}$. It starts by computationally segmenting all structures belonging to a modular enzyme family along structurally conserved sites and assembling the resulting modular fragments to generate a huge combinatorial diversity of backbones. Instead of using the natural sequences of the fragments, as in natural evolution or laboratory genetic recombination ${ }^{39,40}$, we next design the sequence of the entire protein $(>300$ amino acids) to maximize compatibility between the fragments and stabilize the active-site geometry. Each design step, therefore, introduces insertions and deletions as well as dozens of stabilizing mutations. Thus, although our method is inspired by natural evolutionary processes, each step is vastly more radical than individual recombination, insertion, deletion, and mutation events that occur in evolution, in which each event must be at least neutral in fitness or it is likely to be purged ${ }^{37,41}$. Despite having as many as 150 mutations from any natural enzyme, designed enzymes were stable, structurally accurate and highly active without requiring laboratory evolution. The results, therefore, provide proof-of-principle for fully automated design of stable, diverse, and highly active enzymes that catalyze complex reactions.

\section{Results}

The PLL and GH10 families. To test the design method's generality, we targeted two structurally diverse and wellcharacterized TIM-barrel enzyme families exhibiting very different activity profiles: Phosphotriesterase-Like Lactonases (PLL) and Glycoside Hydrolase 10 (GH10) xylanases ${ }^{42}$. PLLs are a group of evolutionarily divergent enzymes ${ }^{43}$ that possess a bimetal center, which activates a water molecule for nucleophilic attack on the activated scissile bond of lactones (Fig. 1a). PLLs have potential applications in bacterial biofilm degradation and in the detoxification of organophosphates, including of highly toxic nerve agents ${ }^{44-47}$. GH10 enzymes hydrolyze the $\beta-1,4$ glycosidic bonds linking the xyloside units that comprise the backbone of the polysaccharide xylan, which is second only to cellulose in abundance in the plant cell wall ${ }^{48}$; xylanases are, therefore, essential for biomass degradation ${ }^{49,50}$. The GH10 catalytic core comprises two proximal and structurally fully conserved Glu sidechains, one acting as the nucleophile, which attacks the glycosidic bond, and the other as the protonating acid ${ }^{51}$ (Fig. 1a). Furthermore, most PLLs are obligate homodimers (Supplementary Fig. 1), while GH10s are monomers, and the PLL family is highly diverse, including members with $<25 \%$ pairwise sequence identity. Thus, the two enzyme families we targeted for design are unrelated in sequence, oligomeric state, active-site structure, and catalytic activity. In both families, the active-site pocket is complex and comprises positions from most $\beta-\alpha$ units (units $2-8$ in GH10s and 1, 4, 5, 6, and 8 in PLLs, Fig. 1b). By contrast to previous enzyme-design studies, both reactions target biological rather than model substrates, and glycosidic-bond hydrolysis involves a high activation barrier, presenting an additional challenge.

In each family, some of the eight $\beta$ - $\alpha$ backbone units are structurally highly diverse (Fig. 1c). Structural diversity presents opportunities for vastly increasing the number of potential backbones for design through combinatorial backbone assembly compared to the limited number of backbones observed in experimentally determined structures (114 and 154 for PLL and GH10, respectively). To estimate the potential for diversifying the backbones through combinatorial backbone assembly, we structurally clustered the eight $\beta-\alpha$ units in the GH10 family by $1 \AA$ root-mean-square deviation (rmsd). We then computed the number of possible combinations, assuming that all $\beta$ - $\alpha$ units could be recombined with all others, yielding a total of $10^{10}$ different backbones, exceeding the number of GH10 structures in the PDB (Protein Database) by eight orders of magnitude. We also reasoned that since all backbone fragments originate from natural enzymes, the likelihood of obtaining stable and functional enzymes from assembly is much higher than using naive scaffold libraries as in past enzyme $\operatorname{design}^{28-30}$ 
a

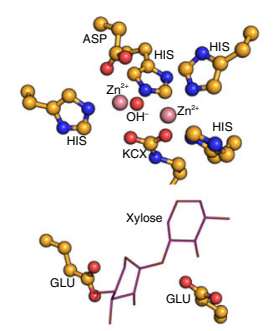

b

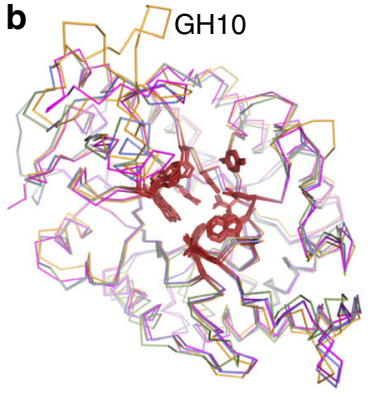

c

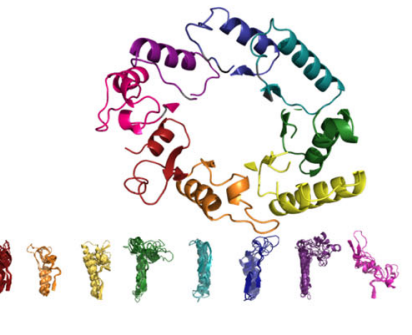

d

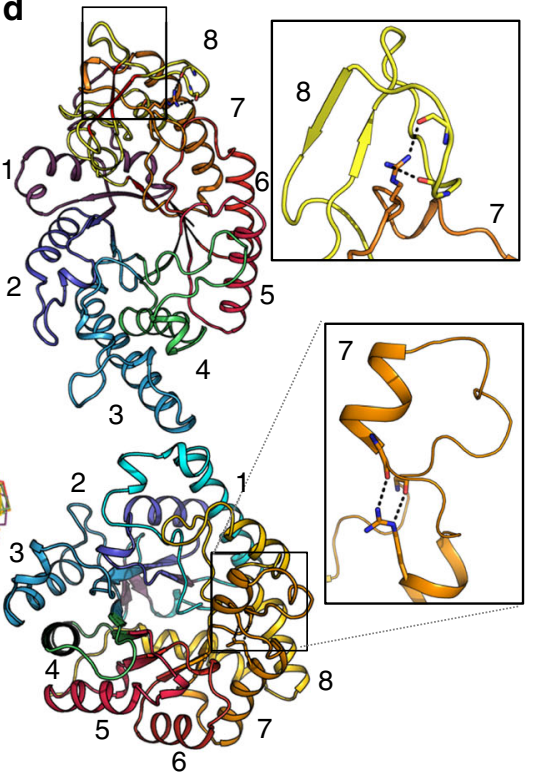

Fig. 1 Combinatorial backbone assembly and design of enzymes. a Active-site constellations of the two targeted enzyme families: (top) PLLs comprising six chelating sidechains (including a carbamylated lysine, KCX), two metal ions, and a hydroxyl ion, and (bottom) GH10s comprising two catalytic Glu sidechains and the substrate. $\mathbf{b}$ Active-site pocket residues that are involved in substrate recognition (red sticks) are held fixed during design. $\mathbf{c}$ In combinatorial backbone assembly, homologous but structurally diverse enzymes are segmented along structurally conserved positions to produce exchangeable backbone fragments (bottom). The fragments are computationally recombined and sequence-optimized to generate new low-energy structures. d Irregular, long-range interactions, including buried charges, stabilize $\beta$ - $\alpha$ backbone conformations. (top) GH10 $\beta-\alpha$ units 7 and 8 (PDB ID: 4PUE). (bottom) The long $\beta-\alpha$ loop (16 amino acids) of the P. diminuta phosphotriesterase (PDB ID: 2R1N)

Combinatorial backbone assembly and design. We segmented the structures in each family according to points of maximal structural conservation in the $\beta$-strands and extracted the backbone conformations of each segment for use in subsequent backbone assembly ${ }^{52}$ (Fig. 1c). The choice of how to segment the backbone into $\beta-\alpha$ units is crucial for design success. Since our method samples backbone fragments independently of one another, each fragment must encode the most important stabilizing contacts; some stabilizing contacts, however, occur between adjacent fragments (Fig. 1d). To test the effects of different segmentation schemes on design success, we chose three segmentations for GH10 designs (Fig. 2): (1) A completely unbiased segmentation, in which each of the eight $\beta$ - $\alpha$ units comprising the TIM-barrel were sampled independently of one another, maximally sampling backbone conformation space (design series xyl8); (2) A structure-based segmentation, comprising four backbone units, each of which forms stabilizing intrasegment contacts: $\beta-\alpha$ units $1,2-4,5-6$, and $7-8$ (series xyl4); and (3) another structure-based though discontinuous segmentation, where the least conformationally diverse segments, $\beta$ - $\alpha$ units 1 and 5-6, formed one constant backbone segment and two other segments were formed by the variable units 2-4 and 7-8 (series xyl3). PLLs, by contrast to GH10s, are obligate homodimers ${ }^{53,54}$. We, therefore, sampled up to five fragments: one comprising the crucial homodimer interface formed by $\beta-\alpha$ units $1-3$ and 8 and up to three other segments from units $4,5,6$, and 7 (design series pll2, pll3, and pll4, which were assembled from 2, 3, or 4 PLL backbones, respectively). Thus, the computational design strategy is amenable to encoding a wide range of constraints inferred from experimental or structural analysis.

To assemble backbones and design new sequences, we generalized the Rosetta AbDesign method-originally developed to design new antibodies from backbone fragments of natural ones $^{52,55,56}$ (Supplementary Fig. 2, Supplementary Movie 1). For each of the segmentation schemes, we started from a random combination of backbone fragments. In each design step, $A b D e-$ sign samples a single backbone fragment from the conformation database and designs the protein's amino acid sequence. Since the entire protein (>300 amino acids) needs to be designed to accommodate the large backbone changes introduced in each step, we used position-specific scoring matrices (PSSMs) to constrain amino acid choices at each position to identities that are commonly observed in a multiple-sequence alignment of natural family members. The PSSMs also focus design calculations on a sequence subspace that is more likely to include stable, folded, and active enzymes. Furthermore, the method does not model the enzyme-transition-state complex, which is often associated with modeling uncertainties and inaccuracies ${ }^{33}$. Instead, residues in direct contact with the bi-metal center in PLLs and the two catalytic Glu residues in GH10 as well as 11 additional residues directly involved in substrate binding, were not allowed to change sidechain conformation during design (Fig. 1b). Following sequence design, the new structure was accepted if it was lower in energy than the previous one, and higher-energy structures were accepted probabilistically. The designs were then ranked by Rosetta energy, clustered by backbone conformation to obtain conformationally unique structures (Supplementary Fig. 3) and subjected to the PROSS stability design algorithm in all regions outside the active-site pockets ${ }^{57}$. PROSS introduced dozens of mutations to each design ( $20 \pm 6$ and $36 \pm 7$ mutations in GH10 and PLL designs, respectively). Visual inspection indicated that the PROSS-designed mutations eliminated core cavities and improved surface polarity and were, therefore, likely to improve protein stability and expressibility. 
a

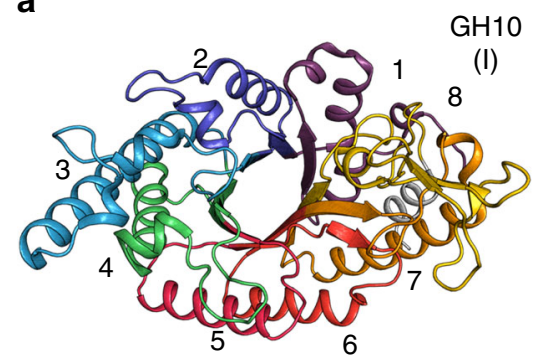

C

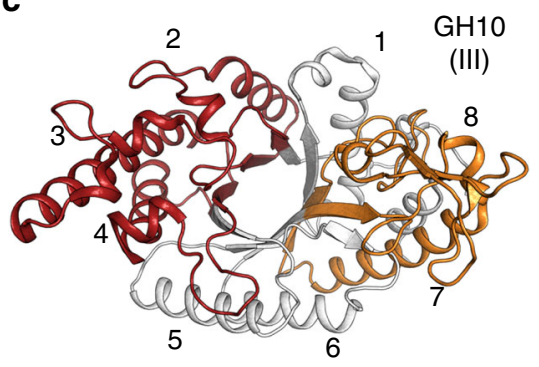

b

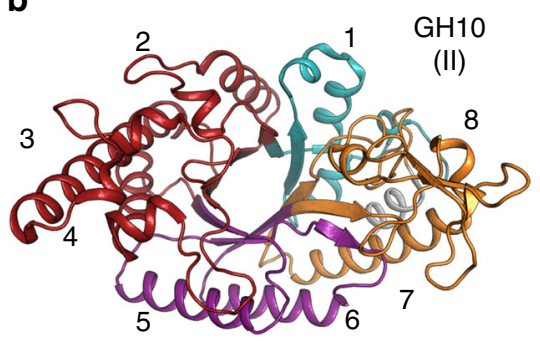

d

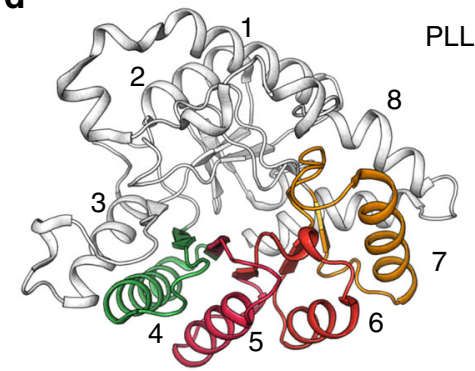

Fig. 2 Different segmentation schemes used in combinatorial backbone assembly. a $\mathrm{GH} 10(\mathrm{I})$ : Each of eight $\beta$ - $\alpha$ units were sampled independently, for maximal backbone diversity. b GH1O(II): Segmenting $\beta-\alpha$ units 1, 2-4, 5-6, and 7-8 to preserve stabilizing interactions within each segment. c GH10(III): a discontinuous segmentation, in which the structurally conserved $\beta-\alpha$ units 1 and $5-6$ formed one segment and two other segments were formed by units 2-4 and 7-8. d The homodimer interface in PLLs ( $\beta-\alpha$ units 1-3 and 8, gray) was used as one backbone segment and units 4-7 were sampled independently

Stable and highly active designs. Synthetic genes encoding 34 PLL and 43 GH10 designs were fused C-terminally to maltosebinding protein (MBP), which served as a solubility and affinitypurification tag. The designs were then overexpressed in E. coli BL21 DE3 cells and purified using an amylose (PLL designs) or Ni-NTA (GH10 designs) column. All the designs expressed solubly, and $>70 \%$ exhibited high expression yields (20-200 mg protein per liter of bacterial culture, Supplementary Fig. 4). Thus, despite as many as 150 mutations relative to any natural enzyme (Table 1, Supplementary Fig. 5), as MBP fusions, the designs did not require iterative rounds of in vitro evolution to optimize expressibility.

We initially screened each of the $43 \mathrm{GH} 10$ designs for xylanase activity with a qualitative assay that measures the formation of reducing sugars released from natural beechwood xylan ${ }^{58}$, finding that 20 of the 43 designs (46\%) were active. We then selected the eight most active designs and two natural GH10 enzymes for quantitative kinetic analysis with the chromogenic substrate 4 -nitrophenyl $\beta$-xylobioside (O-PNPX ${ }_{2}$ ) (Table 1, Supplementary Tables 1 and 2 and Supplementary Fig. 6). The kinetic analysis revealed a wide range of catalytic efficiencies $\left(k_{\text {cat }} / K_{\mathrm{M}}\right)$, and encouragingly, the two most efficient designs, xyl3.1 and xyl3.2, exhibited rates within fivefold of natural GH10 family members (Fig. 3a, b), despite having $>100$ mutations relative to any natural GH10 enzyme (Table 1).

In many industrial applications, GH10 enzymes are subjected to high temperature and acidic $\mathrm{pH}$. Some designs exhibited maximal activity at $45^{\circ} \mathrm{C}$, and some retained full activity even at $50{ }^{\circ} \mathrm{C}$ (Supplementary Fig. 7), with a $\mathrm{pH}$ optimum at 6-6.5 (Supplementary Fig. 8), similar to stability and activity profiles of natural GH10 enzymes. We thus concluded that the automated design method yielded several enzymes that were distant in sequence from any natural enzyme, yet showed similar catalytic and stability profiles to those observed in nature.

Natural PLLs exhibit a range of chemically related hydrolytic activities, including the hydrolysis of lactones, esters, and phosphotriesters. We initially tested the 34 designed PLLs with the artificial substrate 5-thiobutyl butyrolactone (TBBL) ${ }^{59}$, finding seven active designs (Table 1). We subsequently measured the activity of these seven enzymes with a range of substrates: the natural aliphatic $\gamma$-nonanoic lactone, the ester p-nitrophenyl acetate, and the pesticide phosphotriester paraoxon (Supplementary Table 3; Fig. 3c, and Supplementary Figs. 9 and 10). PLL activity was tested with $\mathrm{Co}^{2+}$ or $\mathrm{Zn}^{2+}$, and in most cases much higher activity was observed with $\mathrm{Co}^{2+}$, similar to previous reports ${ }^{60}$. Four PLL designs hydrolyzed the less activated aliphatic $\gamma$-nonanoic lactone, four exhibited esterase activity, and six hydrolyzed the phosphotriester paraoxon. Strikingly, the lactonase and esterase catalytic efficiencies of the four most active PLL designs were similar to those of natural PLLs (Table 1 and Fig. 3c, d). Indeed, design pll2.1 exhibited roughly twofold higher efficiency of TBBL hydrolysis and pll2.4 exhibited an order of magnitude higher efficiency in the hydrolysis of $\gamma$-nonanoic lactone and the pesticide paraoxon than the two natural enzymes. Hence, the designs exhibited features such as high catalytic efficiency and substrate promiscuity, while sampling sequence and conformation space widely (Supplementary Fig. 3). These designs can, therefore, be used as starting points for altering the selectivity profile or discovering new catalytic activities through active-site design or laboratory evolution.

Thermal stability is an essential property of enzymes in many biotechnological applications and low stability often constrains laboratory evolution of new activities ${ }^{41}$. Following overexpression of the active GH10 and PLL designs, we proteolytically cleaved the N-terminal MBP fusion, and subjected the enzymes to thermal denaturation, noting that all designs exhibited high apparent melting temperatures $\left(T_{\mathrm{m}}\right)$ in the range of $50-82{ }^{\circ} \mathrm{C}$ (Table 1 and Supplementary Figs. 11 and 12), comparable to the apparent $T_{\mathrm{m}}$ of natural enzymes in these families, including enzymes from thermophiles.

Atomic precision underlies high catalytic efficiency. The active designs spanned five orders of magnitude in catalytic efficiency. For instance, the most active GH10 design xyl3.1 and the least 


\section{Table 1 Parameters of active GH10 and PLL designs}

\begin{tabular}{|c|c|c|c|}
\hline Design/native enzyme & $\begin{array}{l}\text { Mutations to nearest natural } \\
\text { protein }^{\mathrm{a}}\end{array}$ & $T_{m,}{ }^{\circ} \mathrm{C}$ & $k_{\text {cat }} / K_{M}, M^{-1} s^{-1} b$ \\
\hline xyl3.1 & 105 & 72 & $9417 \pm 311$ \\
\hline xyl3.2 & 112 & 56 & $5060 \pm 280$ \\
\hline xyl3.3 & 137 & 73 & $96 \pm 10$ \\
\hline$x y \mid 4.1$ & 141 & 50 & $36.3 \pm 0.1$ \\
\hline xyl4.2 & 139 & 59 & $1.297 \pm 0.004$ \\
\hline xyl8.1 & 159 & 61 & $156 \pm 4$ \\
\hline xyl8.2 & 121 & 73 & $74 \pm 4$ \\
\hline xyl8.3 & 130 & 57 & $0.61 \pm 0.01$ \\
\hline Xylanase from Geobacillus stearothermophilus (PDB ID: 4PUD) & & $73(78)^{c}$ & $39,700 \pm 3570$ \\
\hline pll2.1 & 80 & $74(71)$ & $\begin{array}{l}556,520 \pm 13,810(31,921 \pm \\
1746)\end{array}$ \\
\hline pll2.2 & 54 & $79(75)$ & $35,000 \pm 780(9944 \pm 173)$ \\
\hline pll2.3 & 75 & $82(56,67,80)$ & $629 \pm 15(275.5 \pm 4.9)$ \\
\hline pll2.4 & 60 & $82(68,81)$ & $48,290 \pm 1160(70.6 \pm 2.8)$ \\
\hline pll2.5 & 67 & $60(57)$ & $7.3 \pm 0.3$ \\
\hline pll3.1 & 73 & $71(68)$ & $667 \pm 70(4.02 \pm 0.06)$ \\
\hline pll4.1 & 85 & $\operatorname{ND}^{d}(54,74)$ & $21,060 \pm 1100(1655 \pm 106)$ \\
\hline PLL from Sulfolobus solfataricus (PDB ID: 2VC7) & & $>100^{80}$ & $\begin{array}{l}210,000 \pm 4447(181,720 \pm \\
3470)\end{array}$ \\
\hline PLL from Geobacillus kaustophilus (PDB ID: 4WVX) & & ND $(55,67,81)$ & $\begin{array}{l}77,370 \pm 2106(9356 \pm \\
1182)\end{array}$ \\
\hline PTE from Pseudomonas diminuta (PDB ID: 1JGM)e & & 52 & 3460 \\
\hline
\end{tabular}
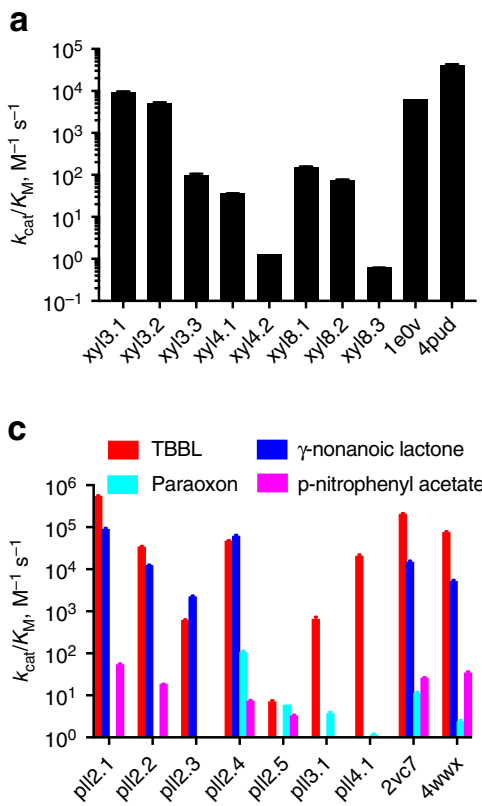

b

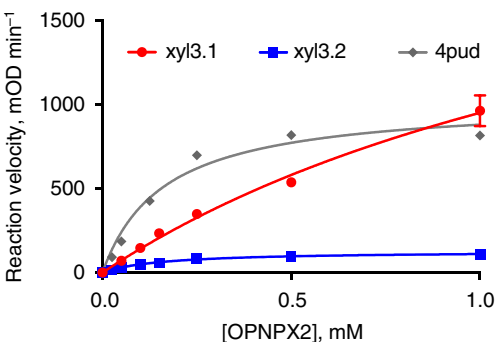

d

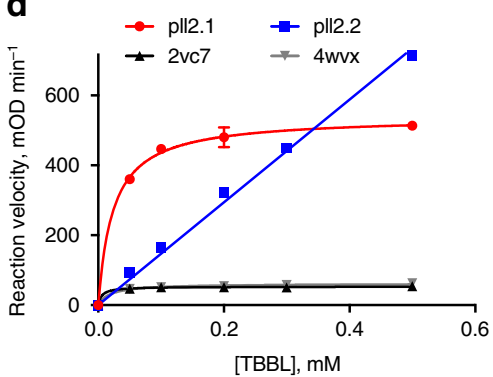

Fig. 3 High catalytic efficiencies in GH10 and PLL designs. a Catalytic efficiency of GH10 designs with OPNPX ${ }_{2}$ compared to natural GH10 enzymes (GH10s from S. lividans and G. stearothermophilus, PDB IDs 1EOV and 4PUD, respectively). Reaction velocities were normalized to $1 \mu \mathrm{M}$ protein. $\mathbf{b}$ Michaelis-Menten curves of the most active GH10 designs relative to a natural GH10 (PDB ID: 4PUD). c Catalytic efficiency of PLL designs with various substrates compared to natural PLLs (from S. solfataricus and G. kaustophilus, PDB IDs 2VC7 and 4WVX, respectively). d Michaelis-Menten curves of the most active PLL designs and natural PLLs with TBBL. Reaction velocities were normalized to $0.1 \mu \mathrm{M}$ protein. Data are the means \pm standard deviation of duplicate reactions 

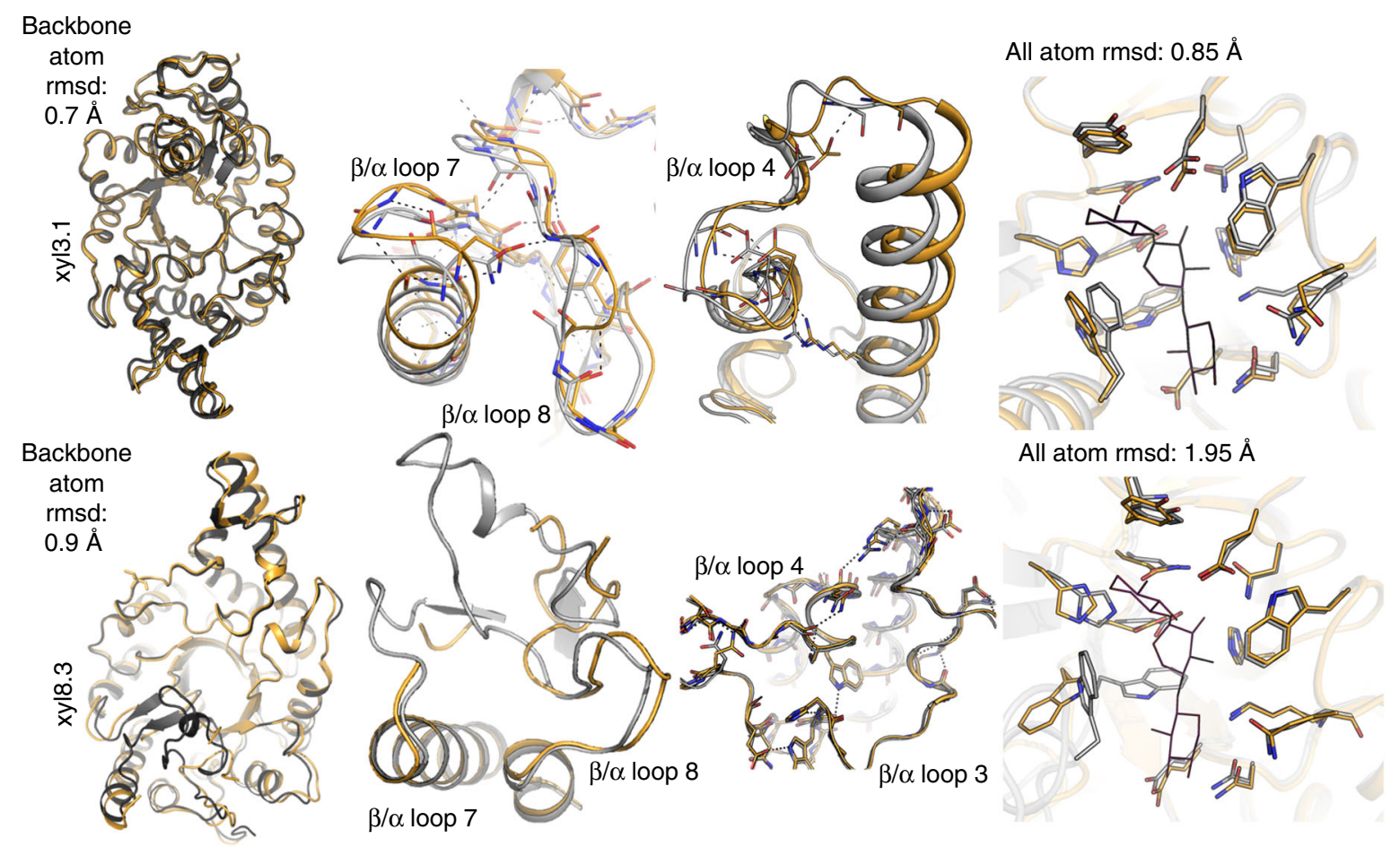

Fig. 4 Design models vs. experimental structures. Shown are design models (gray) and crystal structures (gold) of high and low efficiency GH10 designs (xyl3.1 and xyl8.3, respectively). The designs were crystallized without substrate, and the coordinates of the substrate xylopentaose were extracted from PDB ID 4PUD (thin lines, right-hand side panels) after superimposing the designs' structures. The experimental structure of the high-activity design xyl3.1 is very similar to the design model throughout the backbone, as exemplified in the formation of long-range interactions between $\beta$ - $\alpha$ loops 7 and 8 and within $\beta$ - $\alpha$ segment 4 and in the orientations of all active-site residues. The experimental structure of the low-activity design xyl 8.3 is similar to the model in most segments, for instance, $\beta$ - $\alpha$ segments 3 and 4, and the two catalytic Glu residues, but loops in segments 7 and 8 failed to show substantial electron density

active one xyl8.3 exhibited $\mathrm{OPNPX}_{2}$ hydrolysis efficiencies of 9417 and $0.61 \mathrm{M}^{-1} \mathrm{~s}^{-1}$, respectively. To understand what were the underlying structural reasons for this vast difference in efficiency, we determined their crystallographic structures (Fig. 4, Supplementary Fig. 13, and Supplementary Table 4). In both structures, the two catalytic Glu residues were positioned as in the design conception $(<0.5 \AA$ all-atom root-mean-square deviation (rmsd)). The high-activity design xyl3.1 was also atomically accurate throughout 13 active-site residues that form an intricate hydrogen-bond network surrounding the two catalytic Glu residues $(<1 \AA$ all-atom rmsd), and indeed, across the entire protein, with a total backbone rmsd of $0.7 \AA$. By contrast, the low-activity design xyl8.3 showed conformational changes in the residues that form the active-site hydrogen-bond network ( $2 \AA$ all-atom rmsd). Furthermore, missing electron density in $\beta$ - $\alpha$ loops 7 and 8 suggested that at least parts of the active-site pocket were mobile, although design accuracy was high throughout the segments of the design model and experimental structure that could be aligned ( $0.9 \AA$ backbone rmsd). We, therefore, concluded that accurate positioning of the two catalytic Glu residues was crucial to obtain any level of activity, and that high levels of activity depended on atomic precision and preorganization in a large network of polar residues that surround the catalytic residues.

\section{Discussion}

Combinatorial backbone assembly uses principles inferred from the evolution of enzyme families ${ }^{38}$ : positions at the active-site and ones that are crucial for protein folding and stability are conserved, whereas large backbone and sequence changes, including insertions and deletions, generate vast structural diversity in regions that encode substrate selectivity. Resulting designs were stable and some exhibited atomic accuracy and high catalytic efficiency and promiscuity even with respect to challenging nonactivated substrates that are hydrolyzed by natural members of the respective enzyme families. By contrast, computational design of backbones at enzyme active sites has until now failed to show atomic accuracy, and stability and catalytic efficiencies were $\operatorname{low}^{33,35,61,62}$, limiting the application of computational design to model reactions. It is also notable that expert-guided insertions and deletions at enzyme active sites are challenging and typically require rounds of trial-and-error and optimization, including in the PLLs that were the subject of our study ${ }^{7}$. These laborious iterative strategies can now be bypassed using combinatorial backbone assembly and design. The most active designs were based on fragments from only a few (two or three) different template enzymes, whereas combining fragments from four templates and more generally led to lower efficiency (Fig. 3). It, therefore, appears that future improvements to backbone assembly are needed to fully realize the potential of the design algorithm.

Our method exploits structure and sequence diversity in enzyme families to generate a large combinatorial diversity of backbones, followed by sequence design for stability. The ability demonstrated here to design enzymes exhibiting a large network of active-site residues at atomic accuracy and the resulting high catalytic efficiency greatly simplifies the goal of enzyme design: Instead of depending on accurate transition-state modeling, which, despite recent improvements, still suffers from uncertainty ${ }^{33}$, conserving the natural active-site pocket suffices for wild-type like stability and catalytic efficiency in designs. Our study, therefore, demonstrates a fully automated path to design of structurally diverse enzymes, which catalyze complex reactions, 
despite over 100 mutations from any natural enzyme. The resulting enzymes are stable, active, and highly diverse potential starting points for designing new substrate selectivities, providing an alternative to metagenomic screening and iterative in vitro evolution. An important future direction is to combine backbone fragments from non-homologous families ${ }^{63}$, potentially extending the substrate spectrum beyond that observed within a target enzyme family. Thus, modular backbone assembly and design may provide a path to design of new biocatalysts.

\section{Methods}

A database of natural GH10 and PLL family enzymes. 114 PLL and 154 GH10 structures were downloaded from the Pfam database ${ }^{64,65}$. The structures were segmented along structurally conserved points in the $\beta$-strands. For each segment, the mainchain dihedral angles $(\phi, \psi$, and $\omega)$ and conformation-dependent PSSMs were computed using AbDesign ${ }^{52}$.

Segmentation positions. PLLs were segmented along the following positions (for reference, position numbering is according to the PLL from S. solfataricus, PDB ID: 2VC7): $\beta$ - $\alpha$ unit 4:135-169, 5:171-195, 6:197-217, 7:219-254. GH10 family enzymes were segmented along the following positions (for reference, position numbering is according to GH10 from Thermoanaerobacterium saccharolyticum, PDB ID: 3W24): $\beta-\alpha$ unit 1:20-44, 2:47-83, 3:86-142, 4:145-185, 5:188-218, 6:221-248, 7:251-288, 8:291-319.

Constrained catalytic residues. In PLLs, six metal chelating residues were constrained (for reference, position numbering is according to PLL from S. solfataricus, PDB ID: 2VC7): 22, 24, 137, 170, 199, and 256. In GH10s, the following active-site residues were constrained (for reference, position numbering is according to GH10 from T. saccharolyticum, PDB ID: 3W24): 52, 85, 89, 92, 145, 146, 187, 189, 221, $223,251,292$, and 300.

Combinatorial backbone assembly. For each segmentation scheme, we generated a starting set of 3000 backbone conformations by randomly recombining fragments selected from the conformation databases. The sequence of each design was then optimized using RosettaDesign. From each starting design, 30 steps were taken, in each of which a backbone segment was chosen at random and replaced with a random fragment from the relevant conformation database. Following segment replacement, the sequence of the segment and every residue within $6 \AA$ was designed, and iterations of sidechain packing, backbone and sidechain minimization were conducted to obtain low-energy sequences. The new structure was accepted relative to the previously accepted structure if it passed the Metropolis criterion with a gradually decreasing temperature (simulated annealing Monte Carlo).

Structural clustering of designs. All resulting designs were clustered using MaxCluster (http://www.sbg.bio.ic.ac.uk/maxcluster/).

Stability design. The clustered designs were subjected to the PROSS stability design algorithm ${ }^{57}$, and for each starting design, one stabilized variant (PROSS design variant 6) was selected for experimental characterization.

Rosetta energy function. GH10 designs were computed using the Rosetta Talaris14 all-atom energy function ${ }^{66}$, and PLL designs were computed using the more recent Rosetta energy function REF $15^{67}$. Both energy functions are dominated by all-atom van der Waals packing, hydrogen bonding, electrostatics, and an implicit solvation model.

Sequence identity analysis. We calculated the sequence identity for each of the designed enzymes relative to the closest natural homolog using BLASTP ${ }^{68}$ with the NCBI nonredundant (nr) database (ftp://ftp.ncbi.nlm.nih.gov/blast/db/).

Design sequences. Amino acid sequences of active designs are given in Supplementary Note 1 .

Materials. Paraoxon, p-nitrophenyl acetate, $\gamma$-nonanoic lactone, 5,5-dithio-bis-(2nitrobenzoic acid) (DTNB, Ellman's reagent), m-cresol, and beechwood xylan were purchased from Sigma-Aldrich. TBBL was kindly provided by the Tawfik laboratory ${ }^{59}$. 4-nitrophenyl $\beta$-xylobioside $\left(\mathrm{PNPX}_{2}\right)$ was purchased from Megazyme.

Cloning. Synthetic genes of designs and natural enzymes were codon optimized for efficient $E$. coli expression and custom synthesized as linear fragments by Twist Bioscience. The genes were amplified and cloned into the pETMBPH vector (containing an N-terminal 6-His-tag and $\mathrm{MBP}^{69}$ ) through the EcoRI and PstI restriction sites. The ligated DNA was transformed into E. coli BL21 DE3 cells, and DNA was extracted for Sanger sequencing to validate accuracy. The list of primers used for cloning is given in Supplementary Table 6.

Protein expression and purification. For small-scale expression, $2 \mathrm{ml}$ of $2 \mathrm{YT}$ medium supplemented with $50 \mu \mathrm{g} \mathrm{ml}^{-1}$ kanamycin (and $0.1 \mathrm{mM} \mathrm{ZnCl}_{2}$ or $\mathrm{CoSO}_{4}$ in case of PLLs) were inoculated with a single colony and grown at $37^{\circ} \mathrm{C}$ for $\sim 15 \mathrm{~h}$. In all, $10 \mathrm{ml} 2 \mathrm{YT}$ medium supplemented with $50 \mu \mathrm{g} \mathrm{ml}^{-1}$ kanamycin (and $0.1 \mathrm{mM}$ $\mathrm{ZnCl}_{2}$ or $\mathrm{CoSO}_{4}$ in case of PLLs) were inoculated with $0.2 \mathrm{ml}$ overnight culture and grown at $37^{\circ} \mathrm{C}$ to an $\mathrm{OD}_{600}$ of $\sim 0.6$. Overexpression was induced with $0.2 \mathrm{mM}$ IPTG, and the cultures were grown for $\sim 24 \mathrm{~h}$ at $20^{\circ} \mathrm{C}$. After centrifugation and storage at $-20^{\circ} \mathrm{C}$, the pellets were resuspended in lysis buffer and lysed by sonication.

PLL lysis buffer: $50 \mathrm{mM}$ Tris (pH 8.0), $100 \mathrm{mM} \mathrm{NaCl}, 10 \mathrm{mM} \mathrm{NaHCO} 3,0.1$ $\mathrm{mM} \mathrm{ZnCl}_{2}$ or $\mathrm{CoSO}_{4}$, benzonase and $0.1 \mathrm{mg} \mathrm{ml}^{-1}$ lysozyme.

GH10 lysis buffer: $50 \mathrm{mM}$ Tris ( $\mathrm{pH} 6.8), 100 \mathrm{mM} \mathrm{NaCl}$, benzonase and $0.1 \mathrm{mg} \mathrm{ml}^{-1}$ lysozyme. The PLL proteins were bound to amylose resin (NEB), washed with $50 \mathrm{mM}$ Tris pH 8.0 with $100 \mathrm{mM} \mathrm{NaCl}$ and $0.1 \mathrm{mM} \mathrm{ZnCl}_{2}$ or $\mathrm{CoSO}_{4}$, and eluted with wash buffer containing $10 \mathrm{mM}$ maltose. The GH10 proteins were bound to Ni-NTA resin (Merck), washed with $50 \mathrm{mM}$ Tris pH 6.8 with $100 \mathrm{mM}$ $\mathrm{NaCl}$ and $20 \mathrm{mM}$ imidazole, and eluted with wash buffer containing $250 \mathrm{mM}$ imidazole. Elution fraction was used for SDS-PAGE gel and for initial activity measurements. For further analysis of active designs, the expression was repeated with $50 \mathrm{ml}$ culture, and after purification, the proteins were dialyzed in wash buffer. For crystallization, the expression was performed with $500 \mathrm{ml}$ culture, and after purification, the protein was digested with TEV protease to remove the MBP fusion tag (1:20 TEV, $1 \mathrm{mM}$ DTT, 24-48h at room temperature (RT)). The MBP fusion was removed by binding to Ni-NTA resin, and the protein was purified by gel filtration (HiLoad 26/600 Superdex75 preparative grade column, GE). Protein concentration was estimated by $\mathrm{OD}_{280}$ measurement, and protein expression levels were extrapolated to $\mathrm{mg}$ protein per liter culture.

Preliminary xylanase screening. Xylanase activity was determined qualitatively by measuring the reducing sugars released from xylan by the dinitrosalicylic acid (DNS) method ${ }^{58}$. A typical assay mixture consisted of $20 \mu \mathrm{l}$ citrate buffer $(500 \mathrm{mM}$, $\mathrm{pH} 6.0$ ) added to $80 \mu \mathrm{l}$ cell lysate. The reaction was started by adding $100 \mu \mathrm{l}$ of $2 \%$ beechwood xylan suspended in DDW, and the reaction was continued for $20 \mathrm{~min}$ at $50{ }^{\circ} \mathrm{C}$. The reaction was stopped by transferring the tubes to an ice-water bath One-hundred microliters of the supernatant was then added to $150 \mu \mathrm{DNS}$ reagent, and the tubes were boiled for $10 \mathrm{~min}$, after which the absorbance was measured at $540 \mathrm{~nm}$. The read was compared to a blank sample (cell lysate expressing MBP), and active xylanase designs were taken for further examination.

Kinetic measurements. The kinetic measurements were performed with purified proteins (fused to $\mathrm{MBP}$ ) in activity buffer (PLL: $25^{\circ} \mathrm{C}, 50 \mathrm{mM}$ Tris $\mathrm{pH} 8.0$ with $100 \mathrm{mM} \mathrm{NaCl}$, supplemented with $0.1 \mathrm{mM} \mathrm{ZnCl}_{2}$ or $\mathrm{CoSO}_{4}, \mathrm{GH} 10: 37^{\circ} \mathrm{C}, 50 \mathrm{mM}$ Tris pH 6.5 with $150 \mathrm{mM} \mathrm{NaCl}$ ). A range of enzyme concentrations was used, depending on the activity. The activity of PLLs was tested at $20-22{ }^{\circ} \mathrm{C}$ with TBBL 59 by coupling with DTNB and monitoring the absorbance at $412 \mathrm{~nm}$ ), $\gamma$-nonanoic lactone ( $\mathrm{pH}$-sensitive assay in $2.5 \mathrm{mM}$ bicine $\mathrm{pH} 8.3$, by monitoring the absorbance of $\mathrm{m}$-cresol indicator at $577 \mathrm{~nm}^{70}$ ), paraoxon and p-nitrophenyl acetate (monitoring the absorbance of the leaving group at $405 \mathrm{~nm}$ ). The kinetic measurements were performed in 96-well plates (optical length $-0.5 \mathrm{~cm}$ ), and background hydrolysis rates were subtracted. The activity of GH10s was tested with O-PNPX by monitoring the absorbance of the leaving group at $405 \mathrm{~nm}$. No background

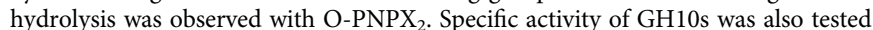
at a range of temperatures $\left(25^{\circ} \mathrm{C}, 37^{\circ} \mathrm{C}, 45^{\circ} \mathrm{C}, 50^{\circ} \mathrm{C}\right.$ ) and at various $\mathrm{pHs}$ (citrate buffer: $\mathrm{pH} 5.0,6.0$, and 6.5 , tris buffer: $\mathrm{pH} 7.0,8.0$, and 9.0).

Determination of kinetic parameters. Kinetic parameters were obtained by fitting the data to the Michaelis-Menten equation $\left[v_{0}=k_{\text {cat }}[\mathrm{E}]_{0}[\mathrm{~S}]_{0} /\left([\mathrm{S}]_{0}+K_{\mathrm{M}}\right)\right]$ using Prism 7. In cases where solubility limited substrate concentrations, data were fitted to the linear regime of the Michaelis-Menten model $\left(v_{0}=[\mathrm{S}]_{0}[\mathrm{E}]_{0} k_{\mathrm{cat}} / K_{\mathrm{M}}\right)$ and $k_{\mathrm{cat}} /$ $K_{\mathrm{M}}$ values were deduced from the slope. The reported values represent the means \pm S.D. of at least two independent measurements.

$\boldsymbol{T}_{\mathbf{m}}$ measurements. $T_{\mathrm{m}}$ measurements were performed after cleavage of the MBP tag from the designs. Two methods were used: ThermoFluor experiments using SYPRO Orange dye (Sigma-Aldrich) on a ViiA 7 real-time PCR machine, with temperature ramp from $25^{\circ} \mathrm{C}$ to $100^{\circ} \mathrm{C}$ at $0.05^{\circ} \mathrm{K} \mathrm{s}^{-1}$ (ref. ${ }^{71}$, and nanoDSF experiments performed on Prometheus ${ }^{\mathrm{mm}}$ NT.Plex instrument (NanoTemper Technologies $)^{72}$. In addition, residual activity of PLL designs was tested following $0.5 \mathrm{~h}$ incubation at various temperatures and cooling to RT.

Structure determination and refinement. Crystals of xyl3.1 and xyl8.3 were obtained using the hanging-drop vapor-diffusion method with a Mosquito robot (TTP LabTech). The crystals of xyl8.3 were grown from 8\% PEG 3500 and $0.05 \mathrm{M}$ Tri-sodium citrate dihydrate $\mathrm{pH}=5.8$. The crystals formed in the space group 
$P 4_{1} 2_{1} 2$, with one complex per asymmetric unit. A complete dataset to $2.1 \AA$ resolution was collected at $100^{\circ} \mathrm{K}$ on a single crystal on in-house RIGAKU RUH3R X-ray. Crystals of xyl3.1 were grown from $0.5 \mathrm{M}\left(\mathrm{NH}_{4}\right)_{2} \mathrm{H}_{2} \mathrm{PO}_{4}$ and $0.05 \mathrm{M}$ sodium acetate $\mathrm{pH}=4.5$. The crystals formed in the space group $\mathrm{H}_{3}$, with one copy per asymmetric unit. A complete dataset to $1.85 \AA$ resolution was collected at $100^{\circ} \mathrm{K}$ on a single crystal on in-house RIGAKU RU-H3R X-ray.

Diffraction images of xyl3.1 and xyl8.3 crystals were indexed and integrated using the Mosflm program ${ }^{73}$, and the integrated reflections were scaled using the SCALA program ${ }^{74}$. Structure factor amplitudes were calculated using TRUNCATE 75 from the CCP4 program suite. xyl3.1 and xyl8.3 structures were solved by molecular replacement with the program PHASER ${ }^{76}$. The models used to solve xyl8.3 and xyl3.1 structures were 3W25 and 3MMD, respectively.

All steps of atomic refinement of both structures were carried out with the CCP4/REFMAC5 program ${ }^{77}$ and by Phenix refine ${ }^{78}$. The models were built into $2 m F_{\text {obs }}-D F_{\text {calc }}$ and $m F_{\text {obs }}-D F_{\text {calc }}$ maps by using the COOT program ${ }^{78,79}$. Details of the refinement statistics of the xyl8.3 and xyl3.1 structures are described in Supplementary Table 4 .

Code availability. All Rosetta design simultations used git version 2c0dc744fb56459daf220abc159f980b1809ecfe of the Rosetta biomolecular modeling software, which is freely available to academics at http://www.rosettacommons. org. The backbone conformation databases and PSSMs are distributed with the Rosetta release. RosettaScripts ${ }^{64}$ and command lines are available in Supplementary Data 1-11.

Data availability. The coordinates of designs xyl8.3 and xyl3.1 are available from the RCSB Protein Data Bank (PDB IDs: 6FHE and 6FHF, respectively). Plasmids encoding the active designs are available from AddGene (IDs 107202-107217). Design protocols are available in the Supplementary Data 12-25. All other data supporting the findings of this study are available from the corresponding author upon reasonable request.

Received: 18 March 2018 Accepted: 13 June 2018

Published online: 17 July 2018

\section{References}

1. Furnham, N. et al. Exploring the evolution of novel enzyme functions within structurally defined protein superfamilies. PLoS Comput. Biol. 8, e1002403 (2012).

2. Orengo, C. A. \& Thornton, J. M. Protein families and their evolution-a structural perspective. Annu. Rev. Biochem. 74, 867-900 (2005).

3. Nagano, N., Orengo, C. A. \& Thornton, J. M. One fold with many functions: the evolutionary relationships between TIM barrel families based on their sequences, structures and functions. J. Mol. Biol. 321, 741-765 (2002).

4. Sterner, R. \& Höcker, B. Catalytic versatility, stability, and evolution of the ( $\beta a)$ 8-barrel enzyme fold. Chem. Rev. 105, 4038-4055 (2005).

5. Lupas, A. N., Ponting, C. P. \& Russell, R. B. On the evolution of protein folds: are similar motifs in different protein folds the result of convergence, insertion, or relics of an ancient peptide world? J. Struct. Biol. 134, 191-203 (2001).

6. Riechmann, L. \& Winter, G. Early protein evolution: building domains from ligand-binding polypeptide segments. J. Mol. Biol. 363, 460-468 (2006).

7. Afriat-Jurnou, L., Jackson, C. J. \& Tawfik, D. S. Reconstructing a missing link in the evolution of a recently diverged phosphotriesterase by active-site loop remodeling. Biochemistry 51, 6047-6055 (2012).

8. Murzin, A. G., Brenner, S. E., Hubbard, T. \& Chothia, C. SCOP: a structural classification of proteins database for the investigation of sequences and structures. J. Mol. Biol. 247, 536-540 (1995).

9. Dellus-Gur, E., Toth-Petroczy, A., Elias, M. \& Tawfik, D. S. What makes a protein fold amenable to functional innovation? fold polarity and stability trade-offs. J. Mol. Biol. 425, 2609-2621 (2013).

10. Arnold, F. H. Directed evolution: Bringing new chemistry to life. Angew. Chem. Int. Ed Engl. 57, 4143-4148 (2017).

11. Goldsmith, M. \& Tawfik, D. S. Enzyme engineering: reaching the maximal catalytic efficiency peak. Curr. Opin. Struct. Biol. 47, 140-150 (2017).

12. Packer, M. S. \& Liu, D. R. Methods for the directed evolution of proteins. Nat. Rev. Genet. 16, 379-394 (2015).

13. Sieber, V., Martinez, C. A. \& Arnold, F. H. Libraries of hybrid proteins from distantly related sequences. Nat. Biotechnol. 19, 456-460 (2001).

14. Meyer, M. M., Hochrein, L. \& Arnold, F. H. Structure-guided SCHEMA recombination of distantly related beta-lactamases. Protein Eng. Des. Sel. 19, 563-570 (2006).

15. Heinzelman, P. et al. A family of thermostable fungal cellulases created by structure-guided recombination. Proc. Natl Acad. Sci. U.S.A. 106, 5610-5615 (2009).
16. Ness, J. E. et al. Synthetic shuffling expands functional protein diversity by allowing amino acids to recombine independently. Nat. Biotechnol. 20, 1251-1255 (2002)

17. Raillard, S. et al. Novel enzyme activities and functional plasticity revealed by recombining highly homologous enzymes. Chem. Biol. 8, 891-898 (2001).

18. Park, H. S. et al. Design and evolution of new catalytic activity with an existing protein scaffold. Science 311, 535-538 (2006).

19. Höcker, B., Claren, J. \& Sterner, R. Mimicking enzyme evolution by generating new ( $\beta \alpha)$ 8-barrels from $(\beta \alpha) 4$-half-barrels. Proc. Natl Acad. Sci. U.S.A. 101, 16448-16453 (2004).

20. Akanuma, S. \& Yamagishi, A. Experimental evidence for the existence of a stable half-barrel subdomain in the (beta/alpha)8-barrel fold. J. Mol. Biol. 382, 458-466 (2008).

21. Bharat, T. A. M., Eisenbeis, S., Zeth, K. \& Hocker, B. A -barrel built by the combination of fragments from different folds. Proc. Natl Acad. Sci. U.S.A. 105, 9942-9947 (2008).

22. Eisenbeis, S. et al. Potential of fragment recombination for rational design of proteins. J. Am. Chem. Soc. 134, 4019-4022 (2012).

23. Offredi, F. et al. De novo backbone and sequence design of an idealized $\alpha / \beta$ barrel protein: Evidence of stable tertiary structure. J. Mol. Biol. 325, 163-174 (2003).

24. Figueroa, M. et al. The unexpected structure of the designed protein Octarellin V.1 forms a challenge for protein structure prediction tools. J. Struct. Biol. 195, 19-30 (2016).

25. Huang, P. -S. et al. De novo design of a four-fold symmetric TIM-barrel protein with atomic-level accuracy. Nat. Chem. Biol. 12, 29-34 (2016).

26. Claren, J., Malisi, C., Hocker, B. \& Sterner, R. Establishing wild-type levels of catalytic activity on natural and artificial $(\beta \alpha) 8$-barrel protein scaffolds. Proc. Natl Acad. Sci. USA 106, 3404-3709 (2009).

27. Sperl, J. M., Rohweder, B., Rajendran, C. \& Sterner, R. Establishing catalytic activity on an artificial $(\beta \alpha) 8$-barrel protein designed from identical halfbarrels. FEBS Lett. 587, 2798-2805 (2013).

28. Jiang, L. et al. De novo computational design of retro-aldol enzymes. Science 319, 1387-1391 (2008).

29. Siegel, J. B. et al. Computational design of an enzyme catalyst for a stereoselective bimolecular Diels-Alder reaction. Science 329, 309-313 (2010).

30. Rothlisberger, D. et al. Kemp elimination catalysts by computational enzyme design. Nature 453, 190-195 (2008).

31. Giger, L. et al. Evolution of a designed retro-aldolase leads to complete active site remodeling. Nat. Chem. Biol. 9, 494 (2013).

32. Khersonsky, O. et al. Bridging the gaps in design methodologies by evolutionary optimization of the stability and proficiency of designed Kemp eliminase KE59. Proc. Natl Acad. Sci. U.S.A. 109, 10358-10363 (2012).

33. Kiss, G., Çelebi-Ölçüm, N., Moretti, R., Baker, D. \& Houk, K. N. Computational enzyme design. Angew. Chem. Int. Ed. Engl. 52, 5700-5725 (2013).

34. Tawfik, D. S. Biochemistry. Loop grafting and the origins of enzyme species. Science 311, 475-476 (2006).

35. Baker, D. An exciting but challenging road ahead for computational enzyme design. Protein Sci. 19, 1817-1819 (2010).

36. Blomberg, R. et al. Precision is essential for efficient catalysis in an evolved Kemp eliminase. Nature 503, 418-421 (2013).

37. Romero, P. A. \& Arnold, F. H. Exploring protein fitness landscapes by directed evolution. Nat. Rev. Mol. Cell Biol. 10, 866-876 (2009).

38. Khersonsky, O. \& Fleishman, S. J. Why reinvent the wheel? Building new proteins based on ready-made parts. Protein Sci. 25, 1179-1187 (2016).

39. Stemmer, W. P. DNA shuffling by random fragmentation and reassembly: in vitro recombination for molecular evolution. Proc. Natl Acad. Sci. U.S.A. 91, 10747-10751 (1994).

40. Voigt, C. A., Martinez, C., Wang, Z.-G., Mayo, S. L. \& Arnold, F. H. Protein building blocks preserved by recombination. Nat. Struct. Biol. 9, 553-558 (2002).

41. Bershtein, S., Segal, M., Bekerman, R., Tokuriki, N. \& Tawfik, D. S. Robustness-epistasis link shapes the fitness landscape of a randomly drifting protein. Nature 444, 929-932 (2006).

42. Lombard, V., Ramulu, H. G., Drula, E., Coutinho, P. M. \& Henrissat, B. The carbohydrate-active enzymes database (CAZy) in 2013. Nucl. Acids Res. 42, D490-D495 (2013).

43. Afriat, L., Roodveldt, C., Manco, G. \& Tawfik, D. S. The latent promiscuity of newly identified microbial lactonases is linked to a recently diverged phosphotriesterase. Biochemistry 45, 13677-13686 (2006).

44. Goldsmith, M. et al. Overcoming an optimization plateau in the directed evolution of highly efficient nerve agent bioscavengers. Protein Eng. Des. Sel. 30, 333-345 (2017).

45. Dumas, D. P., Durst, H. D., Landis, W. G., Raushel, F. M. \& Wild, J. R. Inactivation of organophosphorus nerve agents by the phosphotriesterase from Pseudomonas diminuta. Arch. Biochem. Biophys. 277, 155-159 (1990). 
46. Rémy, B. et al. Harnessing hyperthermostable lactonase from Sulfolobus solfataricus for biotechnological applications. Sci. Rep. 6, 37780 (2016).

47. Hraiech, S. et al. Inhaled lactonase reduces Pseudomonas aeruginosa quorum sensing and mortality in rat pneumonia. PLoS ONE 9, e107125 (2014).

48. McCleary, B. V. \& McGeough, P. A comparison of polysaccharide substrates and reducing sugar methods for the measurement of endo-1,4- $\beta$-xylanase. Appl. Biochem. Biotechnol. 177, 1152-1163 (2015).

49. Bajpai, P. Application of enzymes in the pulp and paper industry. Biotechnol. Prog. 15, 147-157 (1999).

50. Dodd, D. \& Cann, I. K. O. Enzymatic deconstruction of xylan for biofuel production. Glob. Change Biol. Bioenergy 1, 2-17 (2009).

51. Withers, S. G. et al. Direct $1 \mathrm{H}$ n.m.r. determination of the stereochemical course of hydrolyses catalysed by glucanase components of the cellulase complex. Biochem. Biophys. Res. Commun. 139, 487-494 (1986).

52. Lapidoth, G. D. et al. AbDesign: An algorithm for combinatorial backbone design guided by natural conformations and sequences. Proteins 83, 1385-1406 (2015).

53. Hiblot, J., Bzdrenga, J., Champion, C., Chabriere, E. \& Elias, M. Crystal structure of VmoLac, a tentative quorum quenching lactonase from the extremophilic crenarchaeon Vulcanisaeta moutnovskia. Sci. Rep. 5, 8372 (2015).

54. Chow, J. Y. et al. Directed evolution of a thermostable quorum-quenching lactonase from the amidohydrolase superfamily. J. Biol. Chem. 285 , 40911-40920 (2010).

55. Baran, D. et al. Principles for computational design of binding antibodies. Proc. Natl Acad. Sci. U.S.A. 114, 10900-10905 (2017).

56. Khersonsky, O. \& Fleishman, S. J. Incorporating an allosteric regulatory site in an antibody through backbone design. Protein Sci. 26, 807-813 (2017).

57. Goldenzweig, A. et al. Automated structure- and sequence-based design of proteins for high bacterial expression and stability. Mol. Cell 63, 337-346 (2016).

58. Miller, G. L. Use of dinitrosalicylic acid reagent for determination of reducing sugar. Anal. Chem. 31, 426-428 (1959).

59. Khersonsky, O. \& Tawfik, D. S. Chromogenic and fluorogenic assays for the lactonase activity of serum paraoxonases. Chembiochem 7, 49-53 (2006).

60. Elias, M. et al. Structural basis for natural lactonase and promiscuous phosphotriesterase activities. J. Mol. Biol. 379, 1017-1028 (2008).

61. Murphy, P. M., Bolduc, J. M., Gallaher, J. L., Stoddard, B. L. \& Baker, D. Alteration of enzyme specificity by computational loop remodeling and design. Proc. Natl Acad. Sci. U.S.A. 106, 9215-9220 (2009).

62. Bjelic, S. et al. Exploration of alternate catalytic mechanisms and optimization strategies for retroaldolase design. J. Mol. Biol. 426, 256-271 (2014).

63. Höcker, B., Beismann-Driemeyer, S., Hettwer, S., Lustig, A. \& Sterner, R. Dissection of a ( $\beta \alpha)$ 8-barrel enzyme into two folded halves. Nat. Struct. Mol. Biol. 8, 32-36 (2001).

64. Fleishman, S. J. et al. RosettaScripts: a scripting language interface to the Rosetta macromolecular modeling suite. PLoS ONE. 6, e20161 (2011).

65. Finn, R. D. et al. The Pfam protein families database: towards a more sustainable future. Nucl. Acids Res. 44, D279-D285 (2016).

66. Leaver-Fay, A. et al. Scientific benchmarks for guiding macromolecular energy function improvement. Methods Enzymol. 523, 109-143 (2013).

67. Alford, R. F. et al. The Rosetta all-atom energy function for macromolecular modeling and design. J. Chem. Theory Comput. 13, 3031-3048 (2017).

68. Camacho, C. et al. BLAST+: architecture and applications. BMC Bioinforma. 10, 421 (2009).

69. Peleg, Y. \& Unger, T. Application of high-throughput methodologies to the expression of recombinant proteins in E. coli. Methods Mol. Biol. 426, 197-208 (2008).

70. Khersonsky, O. \& Tawfik, D. S. Structure-reactivity studies of serum paraoxonase PON1 suggest that its native activity is lactonase. Biochemistry 44, 6371-6382 (2005).

71. Reinhard, L., Mayerhofer, H., Geerlof, A., Mueller-Dieckmann, J. \& Weiss, M. S. Optimization of protein buffer cocktails using Thermofluor. Acta Crystallogr. Sect. F. Struct. Biol. Cryst. Commun. 69, 209-214 (2013).

72. Lee, E., Badr, M., Lazic, A. \& Duhr, S. Exploring protein stability and aggregation by nanoDSF. Protein Science 25, 104 (2016).
73. Papiz, M. Evolving methods for macromolecular crystallography, 11. Mathematics, Physics and Chemistry-Volume 245, edited by Randy J. Read and Joel Sussman. Crystallography Rev. 15, 123-126 (2009).

74. Evans, P. Scaling and assessment of data quality. Acta Crystallogr. D. Biol. Crystallogr. 62, 72-82 (2005).

75. French, S. \& Wilson, K. On the treatment of negative intensity observations Acta Crystallogr. A. 34, 517-525 (1978).

76. McCoy, A. J. Solving structures of protein complexes by molecular replacement with Phaser. Acta Crystallogr. D. Biol. Crystallogr. 63, 32-41 (2007).

77. Murshudov, G. N., Vagin, A. A. \& Dodson, E. J. Refinement of macromolecular structures by the maximum-likelihood method. Acta Crystallogr. D. Biol. Crystallogr. 53, 240-255 (1997).

78. Afonine, P. V. et al. Towards automated crystallographic structure refinement with phenix.refine. Acta Crystallogr. D. Biol. Crystallogr. 68, 352-367 (2012).

79. Emsley, P. \& Cowtan, K. Coot: model-building tools for molecular graphics. Acta Crystallogr. D. Biol. Crystallogr. 60, 2126-2132 (2004).

80. Hiblot, J., Gotthard, G., Chabriere, E. \& Elias, M. Characterisation of the organophosphate hydrolase catalytic activity of SsoPox. Sci. Rep. 2, 779 (2012).

\section{Acknowledgements}

We thank Melina Shamshoum, Lior Artzi, and Ed Bayer for help in establishing xylanase activity screens in our laboratory, and Nir London, Dan Tawfik, and members of the Fleishman lab for critical reading. The research was supported by a Starting Grant from the European Research Council (335439), the Israel Science Foundation through its Center of Excellence in Structural Cell Biology (1775/12) and its joint India-Israel Research Program (2281/15), and by a charitable donation from Sam Switzer and family.

\section{Author contributions}

G.L., O.K., R.L., and S.J.F. designed the research; G.L. and O.K. contributed equally; G.L. and R.L. developed design methods and designed proteins; G.L. and O.K. performed biochemical experiments; O.D., S.A., and S.R. solved crystal structures; G.L., O.K., and S. J.F. wrote the manuscript with contributions from all authors; and S.J.F supervised the research. All the authors have given approval to the final version of the manuscript.

\section{Additional information}

Supplementary Information accompanies this paper at https://doi.org/10.1038/s41467 018-05205-5.

Competing interests: The authors declare no competing interests.

Reprints and permission information is available online at http://npg.nature.com/ reprintsandpermissions/

Publisher's note: Springer Nature remains neutral with regard to jurisdictional claims in published maps and institutional affiliations.

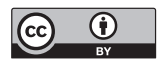

Open Access This article is licensed under a Creative Commons Attribution 4.0 International License, which permits use, sharing, adaptation, distribution and reproduction in any medium or format, as long as you give appropriate credit to the original author(s) and the source, provide a link to the Creative Commons license, and indicate if changes were made. The images or other third party material in this article are included in the article's Creative Commons license, unless indicated otherwise in a credit line to the material. If material is not included in the article's Creative Commons license and your intended use is not permitted by statutory regulation or exceeds the permitted use, you will need to obtain permission directly from the copyright holder. To view a copy of this license, visit http://creativecommons.org/ licenses/by/4.0/.

(c) The Author(s) 2018 